\title{
Optimization of electric vehicle based on triboelectric nanogenerator
}

\author{
Sumit Bhamre ${ }^{l, *}$, Sainath Mali ${ }^{1}$, and Chittaranjan Mane ${ }^{l}$ \\ ${ }^{1}$ JSPM's Jayawantrao Sawant College of Engineering, S. No. 58, Handewadi Road, Hadapsar, Pune 411028, Maharashtra, India.
}

\begin{abstract}
Triboelectric Nanogenerator is a newly invented energy harvesting technology which converts external mechanical energy into electricity based on universally accepted triboelectric principle. In this research paper we have developed an easy way to produce Triboelectric Nanogenerator by using easily available material such as Kapton, Polyethylene Terephthalate (PET Plastic Sheet) and Aluminium Foil unlike more complex material such as Polydimethylsiloxane (PDMS) used by the core researchers. We have also designed and developed a sheet of Triboelectric Nanogenerator and implemented it on electric vehicle and observed the scavenging of friction energy from rolling tyres and also calculated that how much energy Triboelectric Nanogenerator can produce. This successful demonstration of Triboelectric Nanogenerator on wheel and its theoretical calculations provides a promising solution to scavenging the wasted energy of the vehicle and use this wasted energy to increase the battery capacity and range of electric vehicle.
\end{abstract}

\section{Introduction}

Intensive research efforts have been dedicated to the creation of alternative energy technologies due to the rapidly increasing use of fossil fuel and global energy demand in the last decades. The harvesting of environmental mechanical energy is regarded as an effective and promising way to meet energy requirements due to its large abundance and omnipresence. In 2006 a nanogenerator was developed to collect mechanical energy from different mechanical energy sources, including fluid or air flows, ultrasonic waves, deformations, rotations and human activities using nano-composites or piezoelectric nanomaterials. In recent years triboelectric nanogenerator (TENG) has been developed as a new scavenging theory based on the conjunction of triboelectric friction and electric induction

As a power source for a variety of applications, Triboelectric Nanogenerator has quickly shown great promise, including powering of portable electronics, self-allocated sensors, charging lithium ion batteries, electron deposition, electron degradation and electrolyzing water.

The potential of Nano generator to scavenge waste mechanical energy in many different ways is one of the most appealing merits. Friction is a big loss of energy in a rotary mechanism, the most common example being rolling tires on the ground. Studies performed by the US Energy Department showed that the rolling resistance in cars (without including break loss) absorbs 5-7 percent of the energy. In expanding the range of electric vehicles, extracting this type of energy would be promising solution.

\section{Introduction to Triboelectric Nanogenerator}

In 2012, the Zhong Lin Wang group developed the Triboelectric Nanogenerator (TENG) to convert smallscale mechanical energy by a combination of triboelectrification and electrostatic induction into electricity. TENG has unprecedented success and a paradigm shift with a power rating of up to $500 \mathrm{~W} / \mathrm{m} 2$ and an instantaneous conversion efficiency of 70 percent [1]. TENG is lightweight, low density, low cost and can be made with organic materials and neither magnets, nor coils are used for TENG. Most notably, TENG works best with low frequencies $(<5-10 \mathrm{~Hz})$ as opposed to the traditional electromagnetic generator; therefore it is a unique alternative for low frequency body and sea wave (blue energy) energy harvesting.

A Triboelectric Nanogenerator (TENG) can also be utilized to actively detect dynamic and static arising from mechanical agitation using voltage and current output signals respectively.

An energy harvesting device, triboelectric nanogenerator, transforms mechanical external energy into electrical energy through a conjunction of triboelectric effect and electrostatic induction.

In terms of this power generation unit, in the internal circuit, the triboelectric effect generates potential due to a transfer of charging among two fine organic / inorganic films with an opposite tribo-polarity; electrons move

\footnotetext{
* Corresponding author: sumitbbhamre@gmail.com
} 
between two electrodes connected to the back of the films to balance the potentials in the external circuit.

The triboelectric effect is an electrification caused by touch, when a material is electrically charged by the use of friction with a separate material. Triboelectric effect is a common cause of electrostatics every day. In relation to the contact material, the signed charges to be borne by a substance depend on their relative polarity.

This new energy technology offers a number of other benefits, in addition to unparalleled output efficiency, includes low manufacturing and production costs, great strength and reliability and environmental friendliness. The Triboelectric Nanogenerator is ideal for the processing of all kinds of mechanical energy that is available but wasted in our daily lives, including human movement, vibration, mechanical triggers.

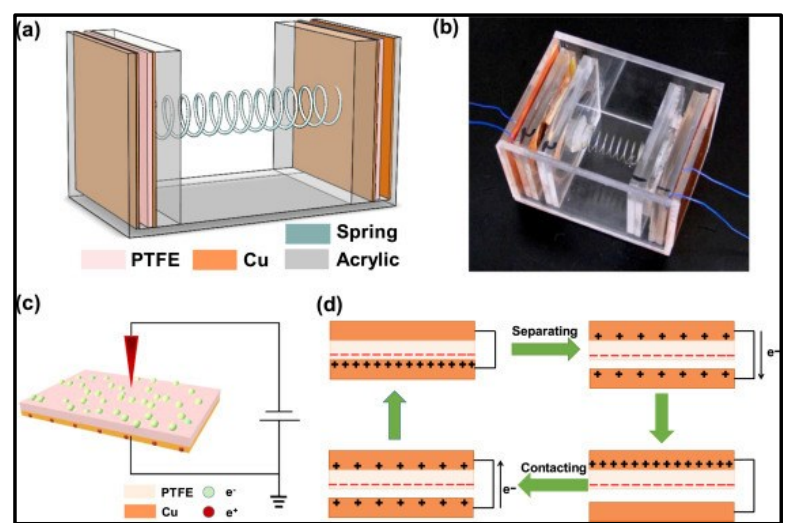

Fig. 1. (a) Illustration of spring type triboelectric nanogenerator (b) A spring-assisted TENG produced computer photograph (c) Schematic method of electron injection to produce negative charges on the PTFE sheet (d) The spring-assisted TENG device's operating principle.

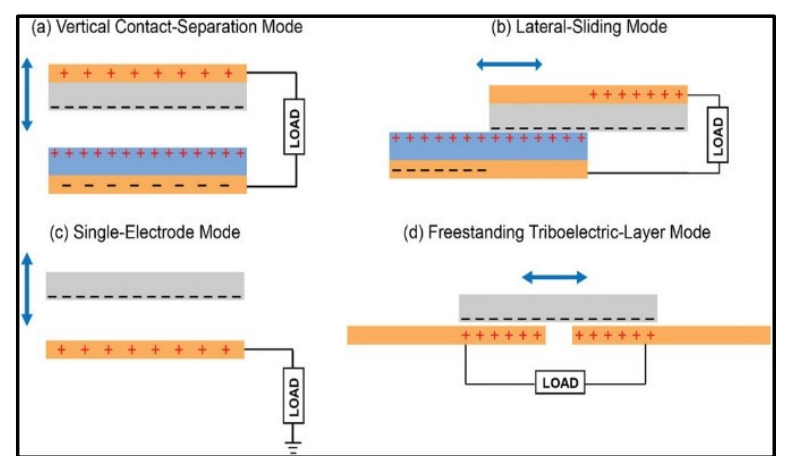

Fig. 2. Types of mode of operating Triboelectric Nanogenerator.

\section{Fabrication of Triboelectric Nanogenerator}

Triboelectric Nanogenerator is a very hard manufacturing process because it requires a piece of sandpaper, then tapped to the bottom of a plastic polystyrene board, then a 10 to 1 ratio of liquid PDMS and cross-connecting system is mixed and then inserted uniformly into the sandpaper. After that a film is taken off the sandpaper and a portion of the $\mathrm{Cu}$ foil is applied to the flat side of the thin film PDMS to conduct pores with a rough surface of thin film and PDMS thin film, serving as a sandpaper electrode [2].

So, to overcome this intricate process we developed a new type of Tribo-generator. We have created it by using materials such as Kapton, Polyethylene Terephthalate (PET Plastic Sheet) and Aluminium Foil. In which Kapton acts as an electron donor, Polyethylene Terephthalate (PET Plastic Sheet) acts as charge acceptor and aluminium acts as charge collector.

Following diagram shows the demonstration of Triboelectric Nanogenerator which we have created;

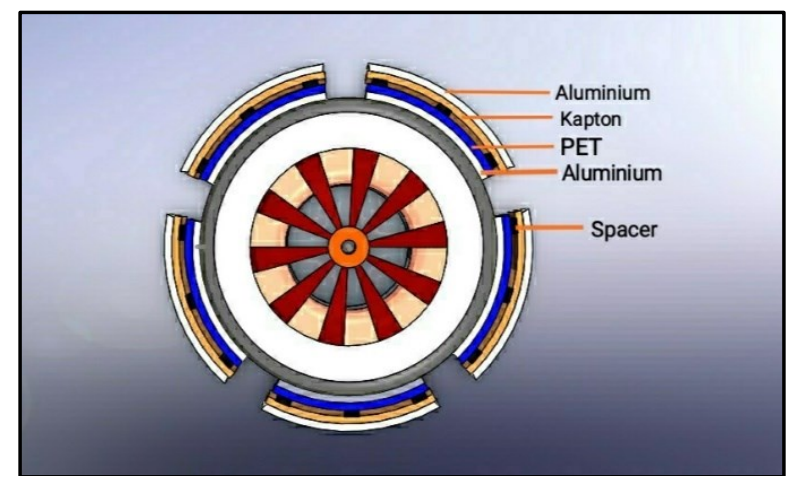

Fig. 3. Implementation of Triboelectric Nanogenerator on wheel.

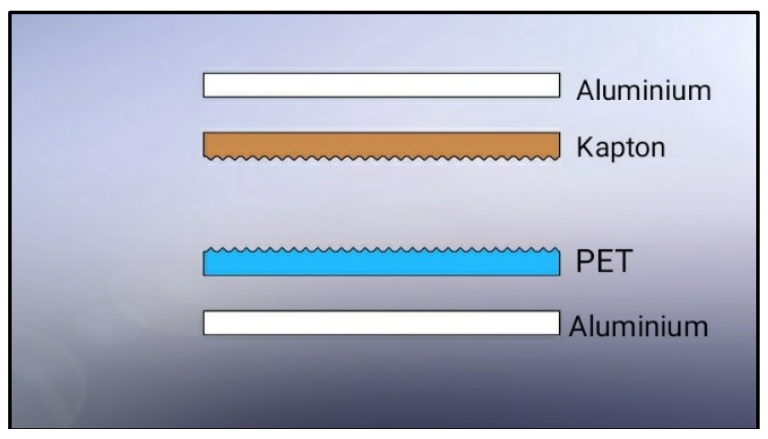

Fig. 4. Two dissimilar material, 1st one created by Aluminium and Kapton and 2nd one created by Polyethylene Terephthalate (PET) and Aluminium.

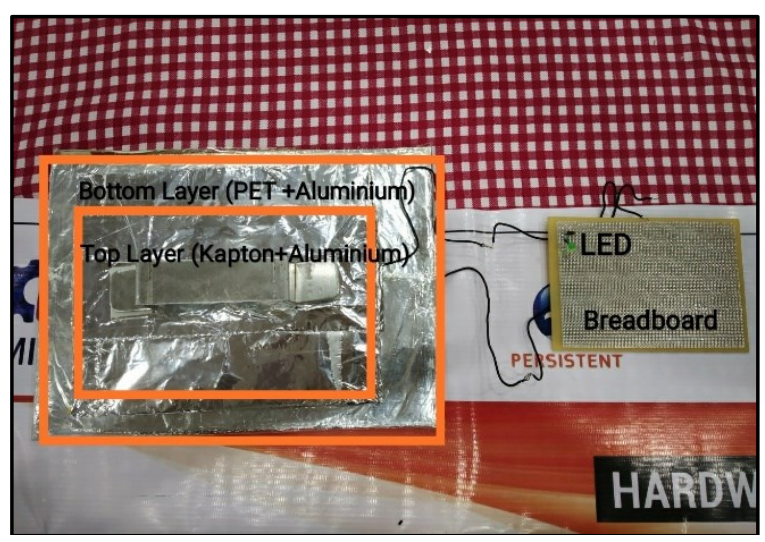

Fig. 5. Actual scale model of the Triboelectric Nanogenerator.

When the 2 dissimilar surface comes in contact and again gets discontent there is simultaneous charge transfer between the two dissimilar materials i.e. in between Kapton and PET, and aluminium acts as an electron charge collector. 
As shown in Fig. 5. The charge collected on the aluminium is transferred to the breadboard and then to the LED which shows the charge transfer circuit completion.

\section{Results and Discussions}

We have taken the results on the digital oscilloscope and every time due to variable load the reading were observed to be different, readings were observed between the range of $5 \mathrm{~V}$ to $16 \mathrm{~V}\left(\mathrm{~V}_{\mathrm{pp}}\right)$, following are the real time graphical demonstration of digital oscilloscope;

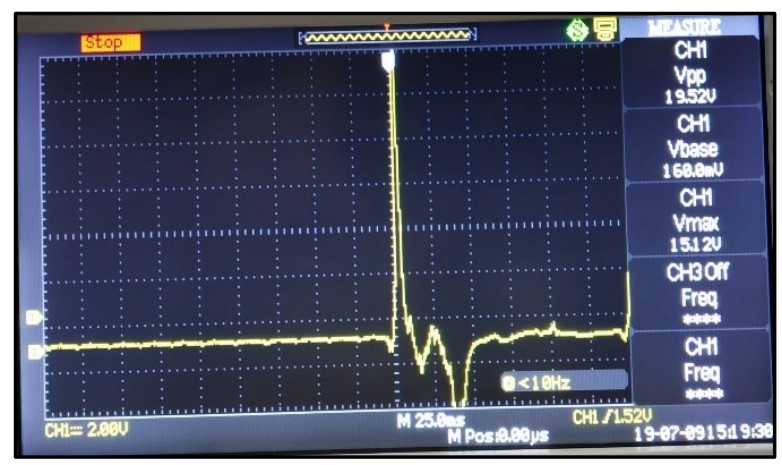

Fig. 6(a). Output result with $V_{p p}$ value $19.52 v$

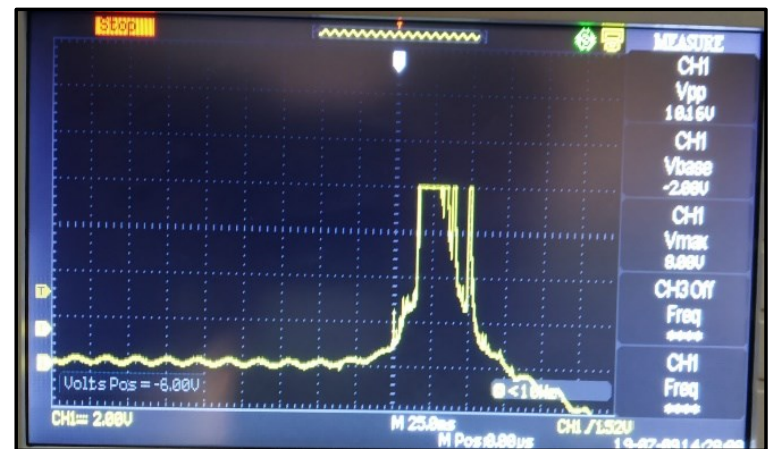

Fig. 6(b). Output result with $V_{p p}$ value $10.16 \mathrm{v}$

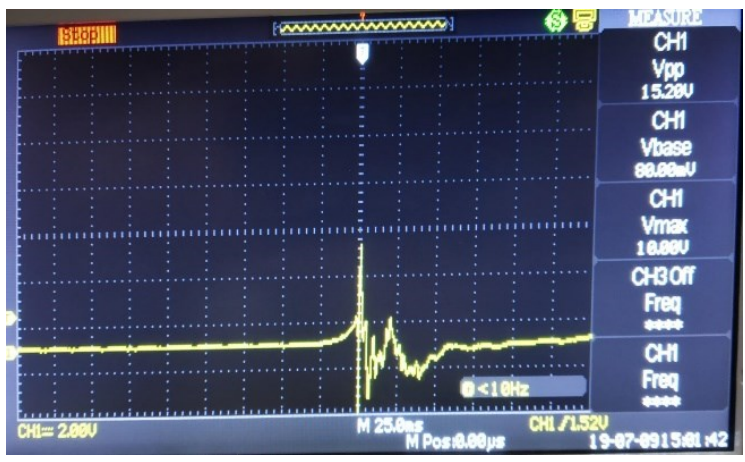

Fig. 6(c). Output result with $V_{p p}$ value $15.20 \mathrm{v}$

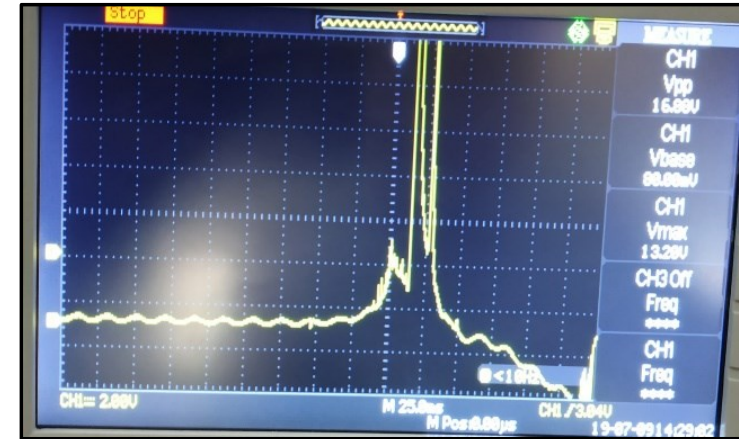

Fig. 6(d). Output result with $V_{p p}$ value $16.88 v$

\section{Calculations}

As of now the maximum instantaneous power output is achieved to be $14.92 \mathrm{~mW}$ [3] by the Triboelectric Nanogenerator and the conversion efficiency is achieved up to $10.4 \%$ [3] of the total energy harvested.

So, assuming that we have implemented these Triboelectric Nano generators on the vehicles tyre as shown in fig. 3, following are the total energy calculation harvested by the 4 wheels in one complete rotation.

\section{Tyre Size: 175/65 R14}

Radius of the tyre: $291.55 \mathrm{~mm}$

Overall width: $174 \mathrm{~mm}$

Now, circumference of the tyre

$=\pi \times \mathrm{D}$

$=\pi \times 583.1$

$=1831.8626 \mathrm{~mm}$

Outer surface area of the tyre $=$ circumference $\mathrm{x}$ width

$=1831.8626 \times 174$

$=318744.1057 \mathrm{~mm}^{2}$

Now let us consider thickness of outer surface be $1 \mathrm{~mm}$;

Therefore, volume of outer surface

$=318744.1057 \mathrm{~mm}^{2} \times 1 \mathrm{~mm}$

$=318744.1057 \mathrm{~mm}^{3}$

Now, it was found that power density of $14.92 \mathrm{~mW} / \mathrm{cm}^{3}$ can be produced with the convergence efficiency of $10.4 \%$

So, the total number of strips can be placed on single tyre

$=318744.1057 \mathrm{~mm}^{3} / 1 \mathrm{~cm}^{3}$

$=318744.1057 \mathrm{~mm}^{3} /(10)^{3} \mathrm{~mm}^{3}$

$=318.7441057$ (Number of strips) 
Therefore, total power produced by 318.744 strips

$=14.92 \mathrm{~mW} \times 318.744$

$=4755.6620 \mathrm{~mW}$

$=4.7556 \mathrm{~W}$

Therefore, power produced by all four tyres in one single revolution is $=4 \times 4.7556 \mathrm{~W}$

$=19.022 \mathrm{~W}$

By considering the conversion efficiency of $10.4 \%$ of the triboelectric Nano generators;

Obtained power

$=19.022 \times(10.4 / 100)$

$=1.9783 \mathrm{~W}$

Hence, the total power output in one revolution of tyre is theoretically obtained to be approximately 2 Watts.

\section{Conclusion}

In brief, we have demonstrated an innovative design of TENG using materials such as Kapton, Polyethylene (PET plastic sheets) and Aluminium based on the triboelectric principle This design has a unique ability to scavenge the friction energy which is generally wasted between rolling wheels and the ground.

The successful modification of Triboelectric Nanogenerator has demonstrated a promising process capability to scavenge the wasted friction energy between the tires and the ground from a running vehicle and thus improves efficiency or the total mileage per charge of electric vehicles.

\section{References}

1. Z.Wang, L.Lin, J. Chen, S. Niu, Y.Zi, Triboelectric Nanogenerators, xxxiii (2016).

2. Y.Mao, D. Geng, E. Liang, X. Wang, Nano Energy, 228 (2015).

3. Y.Mao, D. Geng, E. Liang, X. Wang, Nano Energy, 233 (2015). 